\title{
Research Paper: Effect of Neurofeedback Training on Aggression and Impulsivity in Children With Attention- Deficit/Hyperactivity Disorder: A Double-Blinded Randomized Controlled Trial
}

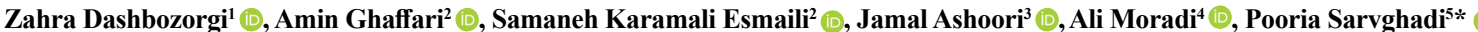 \\ 1. Department of Psychology, Ahvaz Branch, Islamic Azad University, Ahvaz, Iran \\ 2. Department of Occupational Therapy, School of Rehabilitation Science, Iran University of Medical Sciences, Tehran, Iran. \\ 3. Department of Psychology, Isfahan (Khorasgan) Branch, Islamic Azad University. \\ 4. Orthopedic Research Center, Mashhad University of Medical Sciences, Mashhad, Iran. \\ 5. Department of Occupational Therapy, School of Rehabilitation Sciences, Shahid Beheshti University of Medical Sciences, Tehran, Iran.
}

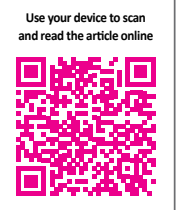

Cittation: Dashbozorgi, Z., Ghaffari, A., Karamali Esmaili, S., Ashoori, J., Moradi, A., \& Sarvghadi, P. (2021). Effect of Neurofeedback Training on Aggression and Impulsivity in Children With Attention-Deficit/Hyperactivity Disorder: A Double-Blinded Randomized Controlled Trial. Basic and Clinical Neuroscience, 12(5), 693-702. http://dx.doi.org/10.32598/bcn.2021.2363.1

http://dx.doi.org/10.32598/bcn.2021.2363.1

\section{(1) (5)}

Article info:

Received: 30 Jan 2020

First Revision: 28 Dec 2020

Accepted: 06 Jan 2021

Available Online: 01 Sep 2021

Keywords:

ADHD, Neurofeedback, Aggression, Impulsivity

\begin{abstract}
$\underline{\text { AB S T RA C T }}$
Introduction: Aggression and impulsivity are some of the behavioral symptoms of AttentionDeficit/Hyperactivity Disorder (ADHD). Neurofeedback (NF) training has been suggested as a promising treatment in these children. This study aimed to investigate the effect of NF training on aggression and impulsivity in schoolchildren with ADHD.

Methods: A total of 40 male elementary school children with ADHD (aged 11.17 \pm 0.97 years) were randomized into the NF and sham groups. The NF group received $12 \mathrm{NF}$ training sessions, each taking about 60 minutes for six consecutive weeks (twice a week), based on the Hammond protocol. The subjects' parents were questioned to evaluate the outcomes, including aggression and impulsivity, using the Buss-Perry Aggression Questionnaire (BPAQ) and Barratt Impulsiveness Scale (BIS).

Results: After the intervention, in the NF group, the BPAQ score changed from $87.60 \pm 9.33$ to $81 \pm 7.23$ and the BIS score from $94.7 \pm 7.25$ to $88.05 \pm 5.4$, which were significant $(\mathrm{P}=0.001)$. The results indicated the large effect size of NF on aggression and impulsivity in ADHD.

Conclusion: Our findings suggest NF training as a clinically applicable method for decreasing aggression and impulsivity, also support concurrent use of medication and NF training in
\end{abstract} children with ADHD.

\section{* Corresponding Author:}

Pooria Sarvghadi, MSc.

Address: Department of Occupational Therapy, School of Rehabilitation Sciences, Shahid Beheshti University of Medical Sciences, Tehran, Iran . Tel: +98 (935) 1697084

E-mail: sarvghadi.pooria@gmail.com 


\section{Highlights}

- Neurofeedback can be a useful method to reduce aggression and impulsivity in ADHD.

- Theta suppression and beta enhancement of brain waves normalize the behavioral symptoms.

- Concomitant use of methylphenidate with neurofeedback results in faster behavioral improvements.

\section{Plain Language Summary}

Neurofeedback is how the brain trains itself by knowing the state of its waves. The brain controls and regulates environmental stimuli based on feedback from its waves. The use of neurofeedback in ADHD is widespread nowadays. Studies have shown a definite effect of neurofeedback on inattention, but there are questions about the impact of this method on other symptoms of the disorder. Children with ADHD usually do not think of the negative, destructive or dangerous consequences of their behaviors, and behaviors are seen as impulsive and aggressive. The present study's findings indicated that this training could help reduce aggression and impulsivity in these children. We concluded that this effect would increase if the medication is taken simultaneously as training. This study was conducted on boys since attention to impulsive and aggressive behaviors is more critical in boys than girls with ADHD. Still, the results can also be generalized to girls. Therefore, parents of children with ADHD can choose neurofeedback training as a therapeutic method to help their children. Since our study did not have a follow-up, the number of sessions should be carefully selected and may require more sessions to sustain changes.

\section{Introduction}

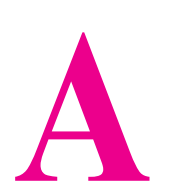

ttention-Deficit/Hyperactivity Disorder (ADHD) is one of the most common childhood developmental disorders with a prevalence of 3.4\% worldwide and 3-5\% in school-age children (American-PsychiatricAssociation, 2013; Polanczyk, Salum, Sugaya, Caye, \& Rohde, 2015). It includes a constant pattern of inattention, hyperactivity/impulsivity, or both, lasting for more than six months (American-Psychiatric-Association, 2013). In addition to core symptoms, aggression as emotional dysregulation and a destructive reaction are comorbid symptoms in ADHD (Harty, Miller, Newcorn, \& Halperin, 2009). Aggression is affected by a wide range of factors, including psychological and genetic factors and neural circuit deficits (Bornmann, Mitelman, \& Beer, 2007; Nelson \& Trainor, 2007). Impulsivity as a component of aggression (Nelson \& Trainor, 2007) also is defined as a tendency towards rapid and unconsidered reactions to internal and external stimulants without caring about the possible unwanted consequences (Sperry, Lynam, Walsh, Horton, \& Kwapil, 2016). Children with ADHD usually represent "impulsive aggression", which is harmful to the individual, society, and public health (Saylor \& Amann, 2016). There is a relationship between deficits in prefrontal and cingulate functional activity with impulsive aggression in ADHD (Puiu et al., 2018). Aggression and impulsivity affect the relation- ship of children, especially boys, with their friends (Zucchetti, Ortega, Scholte, \& Rabaglietti, 2015) and may even affect a child's academic achievement and future employment (Moore et al., 2015). It has been shown that impulsivity could even be associated with aggression in adults with ADHD leading to antisocial outcomes in these people (Comai et al., 2019). Accordingly, determining therapeutic approaches to reduce impulsivity and aggression in ADHD are important issues. Medical treatment is proven to decrease aggression similar to the core symptoms of ADHD (Connor, Glatt, Lopez, Jackson, \& Melloni Jr, 2002). In addition to medical treatments that have side effects, such as decreased appetite, dry mouth, excessive sweating, headache, etc. (Morton and Stockton, 2000), there are non-medical treatments to reduce aggression (Murphy et al., 2014). Among the non-medical treatments for ADHD, Neurofeedback (NF) training is one of the behavioral therapeutic methods that are more efficient than other behavioral approaches, such as cognitive-behavioral therapy (Abbasi, Shariati, \& Tajikzadeh, 2018; Khodabakhsh Pirkalani, Taghavi, \& Rastgoo, 2020; Pendar et al., 2018; Shokhmgar et al., 2019). NF facilitates emotion regulation through operant conditioning and learned control (Enriquez-Geppert, Huster, \& Herrmann, 2017; Sherlin et al., 2011). In this painless noninvasive method, scalp sensors measure and record brain activity, and the patient is taught to self-regulate their dissonant brain waves into normal frequency and rhythm. An increase in theta potency as the prominent 
profile in Electroencephalography (EEG) of children with ADHD has been shown (Chabot and Serfontein, 1996). These findings suggest that suppression of theta and enhancement of beta waves in these children could normalize their EEG and decrease the symptoms, such as aggression and impulsivity.

Many studies have demonstrated the effect of NF on core symptoms of ADHD, especially on impulsivity (Moriyama et al., 2012; Gevensleben et al., 2010; Duric, Assmus, Gundersen, \& Elgen, 2012; Arns, Heinrich $\&$ Strehl, 2014). The meta-analysis of Arns et al. (2014) on randomized controlled trials. Also, van DongenBoomsma, Vollebregt, Slaats-Willemse, and Buitelaar (2013) found that NF did not affect any of the ADHD symptoms. Some studies also have revealed that NF has a positive effect on externalizing behaviors, such as aggression. Konicar et al. (2015) reported that NF training improved severe psychopathic offenders on the behavioral and neurophysiological levels. They discussed that there is a relationship between learned control of the brain and factors that cause aggression. Shanshan and Zichao (2017) indicated that expressive aggression of children with dyslexia decreased resulting from NF. Walker (2013) showed the efficacy of NF on aggression in children and adults with the chief complaint of anger. Only one research reported NF training with a significant effect on reducing the aggression of children with ADHD (Baghban Kazerani, Danesh, \& Abhariyan; 2016). Eliminating or reducing negative behavioral traits, which are clinically challenging and mostly associated with schoolchildren with ADHD, is one of the important goals of treatment (Wilens and Spencer, 2010). The high prevalence of this disorder necessitates a reconsideration of therapeutic methods to help these children. Based on previous studies, in which the effectiveness of NF training as a modern treatment method on behavior has been approved, it has been assumed that its functions in children with ADHD can be evaluated as a non-invasive and safe therapeutic method (Moreno-García, Delgado-Pardo, de Rey, Meneres-Sancho, \& Servera-Barceló, 2015).

As reviewed, studies on the therapeutic effect of NF on impulsivity in ADHD have been controversial and there is still a need for further research (Gevensleben, Rothenberger, Moll, \& Heinrich, 2012; Lofthouse, Arnold, \& Hurt, 2012). The effect of NF on aggression as a consequence of impulsivity has not been sufficiently studied in ADHD. Also, an efficient and reliable clinical protocol for these symptoms is still in question (Yadollahpour, Naraghi-Araani, \& Rashidi, 2015). Therefore, the purpose of the present study was to find out whether NF training is effective on both aggression and impulsivity in a sample of school-aged children with ADHD based on the Hammond Protocol. Given that physical aggression as an outcome of impulsivity is more prevalent in boys than girls (Björkqvist, 2018) and boys with ADHD due to aggression are susceptible to Oppositional Defiant Disorder (ODD) and Conduct Disorder (CD) (Fadus et al., 2020), this study was done on boys with ADHD.

\section{Methods}

\section{Trial design}

In this double-blinded randomized sham group-controlled trial, subjects were selected by convenience sampling using the random number table and randomly assigned to the intervention $(n=20)$ and sham $(n=20)$ groups. Ethical approval number 951342 was obtained from the Mashhad University of Medical Sciences. The study was registered at the Iranian Registry for Clinical Trials (Code: IRCT20160717028964N2). The subjects' parents signed written informed consent with the children's assent.

\section{Participants and settings}

Forty male elementary school children with ADHD were selected from five consulting centers in Pak-dasht (Tehran, Iran) from 2015-2017. The disposition of the sample is presented in Figure 1. The sample size was determined with $90 \%$ test power based on the study of Ghadampour, Shahbazirad, Haghighi Kermanshahi, Mohammadi, and Naseri (2018), which had the closest intervention method to the present study (Ghadampour et al., 2018). Considering $\alpha=0.05$ and $\beta=0.1$, the sample size was estimated to be 16.64 children in each group, which was considered to ensure the sample size for each group of 20 children. The inclusion criteria were the diagnosis of ADHD using The Swanson, Nolan, and Pelham IV scale (SNAP-IV; Parent and Teacher Forms) and the report of a child psychiatrist based on the Diagnostic and Statistical Manual of Mental Disorders, $5^{\text {th }}$ edition (DSM-5) criteria, an IQ $\geq 90$ in Raven's progressive matrices test, studying at public elementary schools, no history of cerebral trauma/injuries, learning disability, and behavioral disorders, taking a stable dose of psychostimulant under the supervision of a child psychiatrist, no history of receiving any other types of non-medical therapies, and the literacy and mental health of parents (based on a score less than 7 on the General Health Questionnaire (GHQ-28)) for answering questions of the measurements. Exclusion criteria included absenteeism for more than two sessions, cancellation of cooperation, and receiving and other treatments and interventions. 


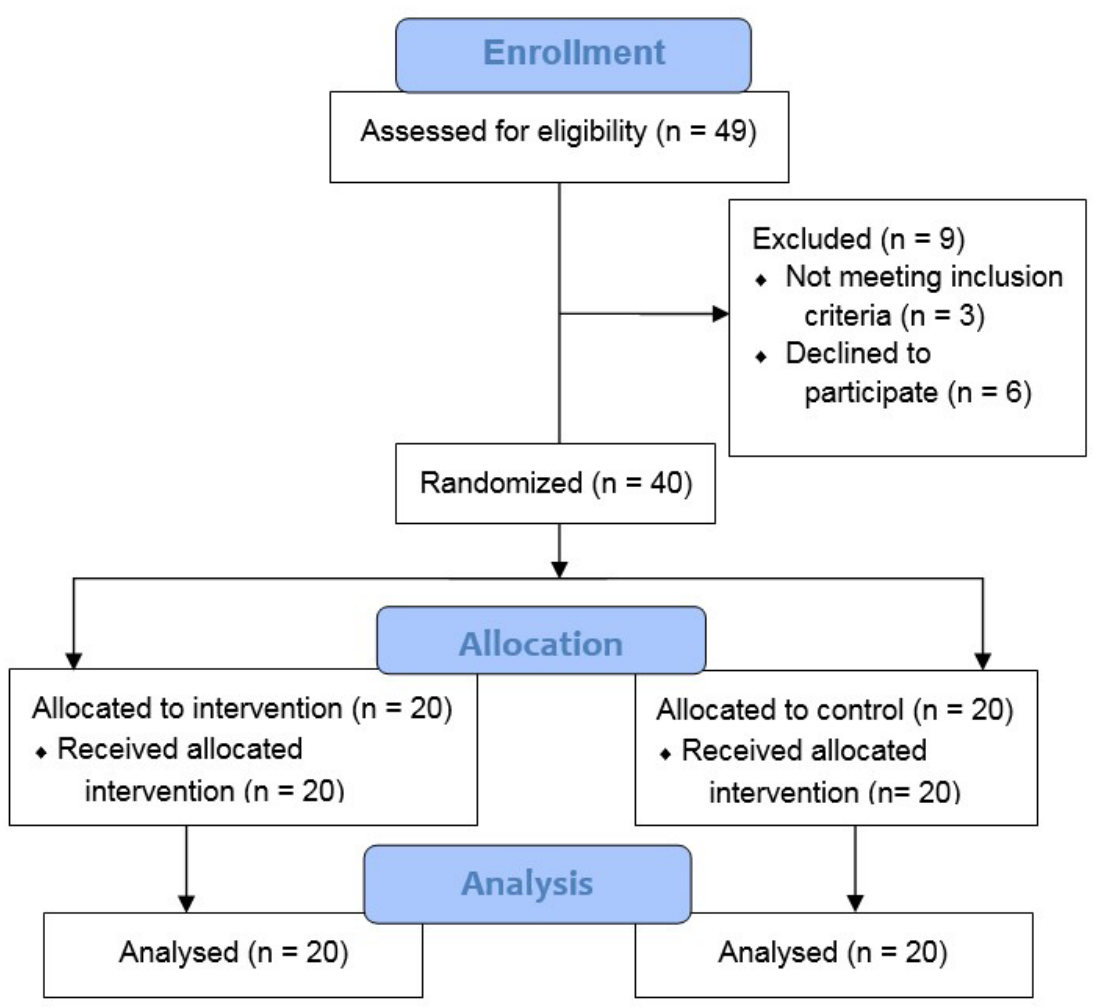

Figure 1. Participants flow

\section{Intervention}

Each student in the experimental group underwent 12 NF training sessions that each took about 60 min for six consecutive weeks (twice a week). A 5-channel ProComp 5 NF system (Canada) with Hammond protocol (a beta/ theta NF treatment protocol with reduction at 4-7 Hz and increment at 15-18 Hz in Q1 and Q2) was used (Sterman \& Egner, 2006). The active, reference, and ground electrodes were placed on Q1, Q2, and right ear, respectively. After a brief demonstration trial, an animation was presented to the subject. Diversion of brain waves from the intended goal resulted in the suspension of the animation. The subject had to regulate their brain waves in the right targeted direction to restart the animation. Each subject was allowed to select his desired animation. The brain practices what is needed to achieve the animation goal through the repetition of training that results in the regulation of brain waves and consequently, treatment. The sham group received no treatment and watched animations that had no therapeutic potency. They waited to receive NF training sessions after the study. NF sessions were performed by two non-blinded neuro therapists. Moreover, the participants were blinded to the study design and group allocation.
Instruments

Raven's progressive matrices test was used to assess IQ as an inclusion criterion. This test consists of sixty matrices that form a logical sequence and are arranged with an increasing degree of difficulty. The test has high validity and reliability in detecting general intelligence (Burke, 1985).

The SNAP-IV scale (parent form) was used to screen ADHD. The questionnaire is an 18-item scale. The first nine questions examine inattentive behaviors, the second nine questions examine hyperactivity/impulsivity symptoms, and all 18 questions identify the combined type of ADHD. The Buss-Perry Aggression Questionnaire (BPAQ) as an outcome measure in this study has 29 items rated using a five-point Likert scale from one to five ( $1=$ strongly disagree to $5=$ strongly agree). A higher score shows higher aggression.

The Barratt Impulsiveness Scale (BIS) was used as an outcome measure. The instrument has 30 items rated using a four-point Likert scale from one to four ( $1=$ rarely or never up to $4=$ almost always). The higher score demonstrates more impulsivity. Patton et al. (1995) have confirmed the convergent validity of the instrument and 
reported its reliability with Cronbach's alpha of 0.83 . The Persian version of this scale has been approved by Javid, Rafiq, Ahmed, \& Khan (2012) with a Cronbach's alpha and test-retest reliability of 0.81 and 0.77 , respectively. Participants accomplished the assessments, and a senior occupational therapist, who was blinded in neurofeedback training, gathered the questionnaires and gave them to a senior biostatistician, who was also blinded to the study.

\section{Statistical analysis}

Data analysis was performed using IBM SPSS version 19. The normal distribution of the data was investigated with the Kolmogorov-Smirnov test. Descriptive statistics, including Means \pm Standard Deviation (SD) and relative frequencies, were used for data analysis for continuous and categorical data, respectively (Mehdizadeh et al., 2019). Multivariate analysis of covariance (MANCOVA) was used to test the statistical assumptions between the groups. The effect sizes were calculated based on the partial eta squared estimation. The values of $.01, .06$, and .14 were calculated as small, medium, and large effects, respectively (Levine and Hullett, 2002; D’Amico, Neilands, \& Zambarano, 2001). The significance level was defined as less than 0.01 .

\section{Results}

The Mean \pm SD age of the subjects in the study was $11.17 \pm 0.97$ years. All subjects received methylphenidate. The baseline characteristics of the groups are shown in Table 1. The assumptions of MANCOVA were investigated. The Kolmogorov-Smirnov test proved the normal distribution of data $(\mathrm{P}=0.200)$. Both Box's $\mathrm{M}(\mathrm{P}=0.115)$ and Levene's tests confirmed homogeneity of co-variances for aggression $(\mathrm{P}=0.807)$ and impulsivity $(0.087)$.
While no significant changes were seen in the pre- and post-test scores of aggression and impulsivity in the sham group, the NF group showed a significant decrease in both aggression and impulsivity scores in the post-test $(\mathrm{P}<0.01)$ (Table 2 and Figure 2).

According to the results in Table 3, the NF group was found with a significant effect on the post-test scores, which considering eta squares, $60.2 \%$ of changes in aggression and $60.9 \%$ of changes in impulsivity were due to the effect of NF training. Therefore, in response to the research question on the effect of NF on aggression and impulsivity, it can be considered that this method significantly reduced both aggression $(\mathrm{F}=54.395 ; \mathrm{P}=0.001)$ and impulsivity $(\mathrm{F}=56.047 ; \mathrm{P}=0.001)$ variables in elementary male students with ADHD. Accordingly, NF training was effective in reducing aggression and impulsivity with a large effect size (Table 3 ).

\section{Discussion}

In this study, the efficacy of NF training was evaluated on decreasing the impulsivity and aggression of male school-aged children with ADHD. Our findings revealed that this intervention could significantly decrease both aggression and impulsivity.

Our findings regarding a reduction in impulsivity as a result of NF training are consistent with those of Arns et al. (2009). By comparing our method with those mentioned, it was found that the number of NF sessions in the present study was less than the others. Given that one of the criteria for inclusion in the present study was receiving a psychostimulant, it seems that NF training in these conditions can lead to greater effects during fewer treatment sessions. In this regard, Zuberer, Ortega, Scholte and Rabaglietti (2018) found that methylphenidate increased learning in the NF sessions. However, van Dongen-Boomsma et al. (2013) concluded that NF

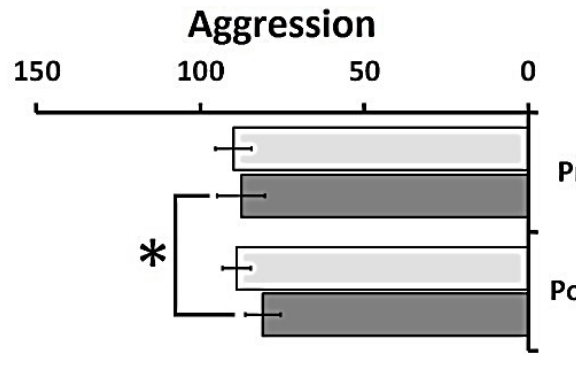

Pre-test

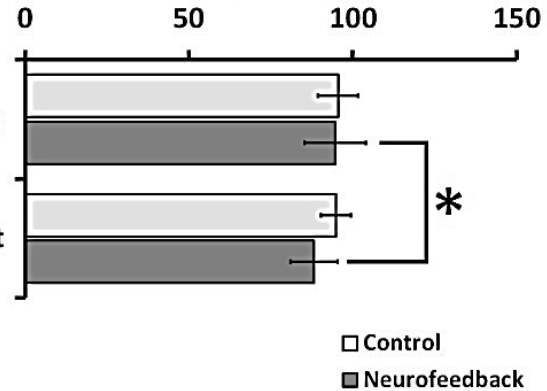

Figure 2. Descriptive statistics of the groups in pre-test and post-test

NEUR SCIENCE 
Table 1. Demographic information of the participants $(n=40)$

\begin{tabular}{ccc}
\hline \multirow{2}{*}{ Variables } & \multicolumn{2}{c}{ Mean \pm SD/No. (\%) } \\
\cline { 2 - 3 } & Neurofeedback Group & Sham Group \\
\hline Age (year) & $11.1 \pm 0.91$ & $11.25 \pm 1.01$ \\
Gender: Male & $20(100)$ & $20(100)$ \\
Grade & $8(40)$ & $7(35)$ \\
Forth Fifth & $5(25)$ & $(30)$ \\
sixth & $7(35)$ & $(35)$ \\
\hline
\end{tabular}

NEUR SCIENCE

did not affect the ADHD symptoms. In their study, all samples did not receive the drug so that more subjects in the control group (3/4) than the NF group (1/2) received methylphenidate (van DongenBoomsma et al., 2013). Perhaps this was a reason for the lack of impact of NF in their study. While in the current study, all subjects were treated with methylphenidate.

The previous studies on different samples showed that NF training is an effective therapeutic method for aggression as an unpleasant behavioral problem (Behboodi et al., 2015, Wilson, 2013; Yaghoobi, 2011; Walker, 2013; Shanshan \& Zichao, 2017; Konicar et al., 2015). However, a few studies have been conducted on the effect of NF on aggression in children with ADHD. The results of the only relevant study were consistent with our results. Kazerani, Talebi, and Gilasian (2013) approved improvement in aggression in children with ADHD resulting from NF training. There were some differences between their study and the present study. In their study, the number of sessions was 30 and the subjects received no medication. Based on our findings and those of $\mathrm{Zu}-$

Table 2. Descriptive statistics of the groups in pre-test and post-test

\begin{tabular}{|c|c|c|c|c|}
\hline \multirow{2}{*}{ Scale } & \multirow{2}{*}{ Group } & \multicolumn{2}{|c|}{ Mean士SD } & \multirow{2}{*}{$\begin{array}{c}\text { Mean } \\
\text { Difference }\end{array}$} \\
\hline & & Pre-test & Post-test & \\
\hline $\begin{array}{l}\text { The Buss- } \\
\text { Perry } \\
\text { Aggression }\end{array}$ & $\begin{array}{l}\text { Neurofeedback group } \\
\qquad(\mathrm{n}=20)\end{array}$ & $87.60 \pm 9.33$ & $81.00 \pm 7.23$ & 6.6 \\
\hline $\begin{array}{l}\text { Questionnaire } \\
\text { (BPAQ) }\end{array}$ & $\begin{array}{l}\text { Sham group } \\
\qquad(n=20)\end{array}$ & $89.85 \pm 5.57$ & $88.85 \pm 4.29$ & 1 \\
\hline $\begin{array}{l}\text { The Barratt } \\
\text { Impulsiveness }\end{array}$ & $\begin{array}{l}\text { Neurofeedback group } \\
\qquad(\mathrm{n}=20)\end{array}$ & $94.70 \pm 7.25$ & $88.05 \pm 5.40$ & 6.65 \\
\hline Scale (BIS) & $\begin{array}{l}\text { Sham group } \\
(n=20)\end{array}$ & $95.50 \pm 6.16$ & $94.85 \pm 4.48$ & 0.65 \\
\hline
\end{tabular}

NEUR SCIENCE

Table 3. Results of the multivariate analysis of covariance between groups

\begin{tabular}{ccccccc}
\hline Scale & Source & $\begin{array}{c}\text { Sum of } \\
\text { Squares }\end{array}$ & $\begin{array}{c}\text { Degree of } \\
\text { Freedom }\end{array}$ & F & P & $\begin{array}{c}\text { Partial Eta } \\
\text { Square }\end{array}$ \\
\hline $\begin{array}{c}\text { The Buss-Perry } \\
\text { Aggression } \\
\text { Questionnaire } \\
\text { (BPAQ) }\end{array}$ & Pre-test & 1092.227 & 1 & 156.236 & 0.001 & 0.813 \\
$\begin{array}{c}\text { The Barratt } \\
\text { Impulsiveness } \\
\text { Scale (BIS) }\end{array}$ & Group & 380.270 & 1 & 54.395 & 0.001 & 0.602 \\
\hline
\end{tabular}

Error 248.26636 
berer et al. (2018), fewer NF sessions will need if children with ADHD receive methylphenidate.

It seems that the Hammond protocol could be useful for children with ADHD. Consistent with this, Bridges (2018) recommended that behavioral symptoms of ADHD were correlated with coherence values within beta and theta bands. Duric et al. (2012) confirmed that enhancement of beta $(15-18 \mathrm{~Hz})$ and suppression of theta $(4-7 \mathrm{~Hz})$ activity have a positive effect on children with ADHD. As an explanation, the effect of a reduction or an increase in the amplitude of brain waves (particularly $4-7 \mathrm{~Hz}$ and $15-18 \mathrm{~Hz}$ ) can improve higher mental functions. NF training facilitates autoregulatory mechanisms, which play essential roles in its normal function (Demos, 2005; Sarvghadi, Ghaffari, \& Rostami, 2019) and increase the ability to inhibit prepotent responses (Baumeister et al., 2018). These positive effects of NF can likely be attributed to the high relevance of neuronal oscillations for complex cognitive processes (Singer, 2018). In this regard, especially medial frontal theta oscillations have been demonstrated to reflect an important mechanism mediating cognitive control (Cavanagh \& Frank, 2014). This training system persuades the brain to correct, modify, and maintain its optimal function through feedback on what has been done and how the normal bioelectrical rhythms have been worked during the last few seconds in an NF session; therefore, the brain is trained to enhance some and suppress some other waves. This practice for the brain eventually might alleviate aggression and impulsivity.

On the other hand, in an NF session, based on the operant conditioning theory (Sherlin et al., 2011), the change in the stimulus (amplitude of brain waves) will result in the predetermined favorable outcome (moving the animation or developing a voice) and learning (self-regulation) will happen. As a result of this process, a child with ADHD learns to change towards optimal behavior, which in turn reduces aggression and impulsivity.

As a study limitation, generalizing the findings of the study would be impossible as a result of the restricted sample size and follow-up deficiency, which was also a considerable hurdle in evaluating the consistency of findings. Making a comparison between NF training and other therapeutic methods, like pharmaceutical and behavioral should be taken into account. Gender discrepancy may also elucidate more information.

\section{Ethical Considerations}

\section{Compliance with ethical guidelines}

This study was approved by the Ethics Committee of the Mashhad University of Medical Sciences (Code: IR.MUMS.FM.REC.1396.548 ).

\section{Funding}

This project was supported by the Mashhad University of Medical Sciences (Grant number: 951342).

\section{Authors' contributions}

All authors equally contributed to preparing this article.

\section{Conflict of interest}

The authors declared no conflicts of interests.

\section{References}

Abbasi, F., Shariati, K., \& Tajikzadeh, F. (2018). Comparison of the effectiveness of cognitive behavioral therapy and neurofeedback: Reducing anxiety symptoms. Archives of Neuroscience, 5(3), e62341. [DOI:10.5812/archneurosci.62341]

American Psychiatric Association. (2013). Diagnostic and statistical manual of mental disorders. Washington, DC: American Psychiatric Association Publishing. [DOI:10.1176/appi. books.9780890425596]

Arns, M., Heinrich, H. \& Strehl, U. (2014). Evaluation of neurofeedback in ADHD: The long and winding road. Biological Psychology, 95, 108-15. [DOI:10.1016/j.biopsycho.2013.11.013] [PMID]

Baghban Kazerani, A., Danesh, E., \& Hasani Abhariyan, P. (2016). [The effectiveness of neurofeedback treatment on aggression reduction in children with Attention Deficit Hyperactivity Disorder (ADHD) (Persian)]. Journal of Neuropsychology, 1(3), 38-56. http://clpsy.journals.pnu.ac.ir/article_3034. html

Baumeister, S., Wolf, I., Holz, N., Boecker-Schlier, R., Adamo, N., \& Holtmann, M., et al. (2018). Neurofeedback training effects on inhibitory brain activation in ADHD: A matter of learning? Neuroscience, 378, 89-99. [DOI:10.1016/j.neuroscience.2016.09.025] [PMID]

Behboodi, M., Shah-Abadi, S., Ansari-Moghaddam, A., Ahmadi, N., \& Shamohammadi, M. (2015). [Investigation efficacy of neurofeedback intervention on reduction aggression behaviors in adolescents (Persian)]. Journal of Modern Rehabilitation 9(2), 62-8. http:// mrj.tums.ac.ir/article-1-5269-en.html

Björkqvist, K. (2018). Gender differences in aggression. Current Opinion in Psychology, 19, 39-42. [DOI:10.1016/j.copsус.2017.03.030] [PMID] 
Bornmann, B. A., Mitelman, S. A., \& Beer, D. A. (2007). Psychotherapeutic relaxation: How it relates to levels of aggression in a school within inpatient child psychiatry: A pilot study. The Arts in Psychotherapy, 34(3), 216-22. [DOI:10.1016/j. aip.2007.01.004]

Burke, H. R. (1985). Raven's Progressive matrices (1938): More on norms, reliability, and validity. Journal of Clinical Psychology, 41(2), 231-5. [DOI:10.1002/1097-4679(198503)41:2<231::AIDJCLP2270410216>3.0.CO;2-Z]

Cavanagh, J. F., \& Frank, M. J. (2014). Frontal theta as a mechanism for cognitive control. Trends in Cognitive Sciences, 18(8), 414-21. [DOI:10.1016/j.tics.2014.04.012] [PMID] [PMCID]

Chabot, R. J., \& Serfontein, G. (1996). Quantitative electroencephalographic profiles of children with attention deficit disorder. Biological Psychiatry, 40(10), 951-63. [DOI:10.1016/00063223(95)00576-5]

Comai, S., Bertazzo, A., Vachon, J., Daigle, M., Toupin, J., \& Côté, G., et al. (2019). Trace elements among a sample of prisoners with mental and personality disorders and aggression: Correlation with impulsivity and ADHD indices. Journal of Trace Elements in Medicine and Biology, 51, 123-9. [DOI:10.1016/j. jtemb.2018.10.008] [PMID]

Connor, D. F., Glatt, S. J., Lopez, I. D., Jackson, D., \& Melloni Jr, R. H. (2002). Psychopharmacology and aggression. I: A metaanalysis of stimulant effects on overt/covert aggression-related behaviors in ADHD. Journal of the American Academy of Child $\mathcal{E}$ Adolescent Psychiatry, 41(3), 253-61. [DOI:10.1097/00004583200203000-00004] [PMID]

D'Amico, E. J., Neilands, T. B., \& Zambarano, R. (2001). Power analysis for multivariate and repeated measures designs: A flexible approach using the SPSS MANOVA procedure. Behavior Research Methods, Instruments, \& Computers, 33(4), 47984. [DOI:10.3758/BF03195405] [PMID]

Demos, J. N. (2005). Getting started with neurofeedback. New York: W. W. Norton \& Company. https://books.google.com/ books?id=EzMhV3EpdqEC\&dq

Duric, N. S., Assmus, J., Gundersen, D., \& Elgen, I. B. (2012). Neurofeedback for the treatment of children and adolescents with ADHD: A randomized and controlled clinical trial using parental reports. BMC Psychiatry, 12, 107. [DOI:10.1186/1471244X-12-107] [PMID] [PMCID]

Enriquez-Geppert, S., Huster, R. J., \& Herrmann, C. S. (2017). EEG-Neurofeedback as a tool to modulate cognition and behavior: A review tutorial. Frontiers in Human Neuroscience, 11, 51. [DOI:10.3389/fnhum.2017.00051] [PMID] [PMCID]

Fadus, M. C., Ginsburg, K. R., Sobowale, K., Halliday-Boykins, C. A., Bryant, B. E., \& Gray, K. M., et al. (2020). Unconscious bias and the diagnosis of disruptive behavior disorders and ADHD in African American and hispanic youth. Academic Psychiatry, 44(1), 95-102. [DOI:10.1007/s40596-019-01127-6] [PMID] [PMCID]

Gevensleben, H., Holl, B., Albrecht, B., Schlamp, D., Kratz, O., \& Studer, P., et al. (2010). Neurofeedback training in children with ADHD: 6-month follow-up of a randomised controlled trial. European Child \& Adolescent Psychiatry, 19(9), 715-24. [DOI:10.1007/s00787-010-0109-5] [PMID] [PMCID]

Gevensleben, H., Rothenberger, A., Moll, G. H., \& Heinrich, H. (2012). Neurofeedback in children with ADHD: Validation and challenges. Expert Review of Neurotherapeutics, 12(4), 44760. [DOI:10.1586/ern.12.22] [PMID]

Ghadampour, E., Shahbazirad, A., Haghighi Kermanshahi, M., Mohammadi, F., \& Naseri, N. (2018). [The effects of sand play therapy in reduction of impulsivity and attention deficit in boys with ADHD (Persian)]. Quarterly Journal of Child Mental Health, 5(2), 36-46. http://childmentalhealth.ir/article1-306-en.html

Javed, M., Rafiq, M., Ahmed, M., \& Khan, M. (2012). Impact of HR practices on employee job satisfaction in public sector organizations of Pakistan. Interdisciplinary Journal of Contemporary Research in Business, 4(1), 348-63. https://www. researchgate.net/publication/256060328_Impact_of_HR_ Practices_on_Employee_Job_Satisfaction_in_Public_Sector_ Organizations_of_Pakistan

Harty, S. C., Miller, C. J., Newcorn, J. H., \& Halperin, J. M. (2009) Adolescents with childhood ADHD and comorbid disruptive behavior disorders: Aggression, anger, and hostility. Child Psychiatry and Human Development, 40(1), 85-97. [DOI:10.1007/ s10578-008-0110-0] [PMID] [PMCID]

Kazerani, F., Talebi, A. A., \& Gilasian, E. (2013). An annotated checklist of the subfamily Syrphinae (Diptera: Syrphidae) of Iran. Entomofauna, 34(33), 409-48. https://www.researchgate. net/profile/Farzaneh-Kazerani/publication/263878023

Khodabakhsh Pirkalani, R., Taghavi, K. S., \& Rastgoo, F. (2020). [Comparison of the effectiveness of cognitive-behavioral therapy and neurofeedback techniques on women's depression adult (Persian)]. Clinical Psychology Studies, 9(36), 25-46. [DOI:10.22054/JCPS.2020.44110.2162]

Konicar, L., Veit, R., Eisenbarth, H., Barth, B., Tonin, P., \& Strehl, U., et al. (2015). Brain self-regulation in criminal psychopaths. Scientific Reports, 5, 9426. [DOI:10.1038/srep09426] [PMID] [PMCID]

Levine, T. R., \& Hullett, C. R. (2002). Eta squared, partial eta squared, and misreporting of effect size in communication research. Human Communication Research, 28(4), 612-25. [DOI:10.1111/j.1468-2958.2002.tb00828.x]

Lofthouse, N., Arnold, L. E., \& Hurt, E. (2012). Current status of neurofeedback for attention-deficit/hyperactivity disorder. Current Psychiatry Reports, 14(5), 536-42. [DOI:10.1007/s11920012-0301-z] [PMID]

Mehdizadeh, M., Martinez-Martin, P., Habibi, S. A. H., Fereshtehnejad, S. M., Abasi, A. A., \& Niazi Khatoon, J., et al. (2019). Reliability and validity of fall efficacy scaleinternational in people with Parkinson's disease during on- and off-drug phases. Parkinson's Disease, 2019, 6505232 [DOI:10.1155/2019/6505232] [PMID] [PMCID]

Moore, S. E., Scott, J. G., Thomas, H. J., Sly, P. D., Whitehouse, A. J. O., \& Zubrick, S. R., et al. (2015). Impact of adolescent peer aggression on later educational and employment outcomes in an Australian cohort. Journal of Adolescence, 43, 39-49. [DOI:10.1016/j.adolescence.2015.05.007] [PMID]

Moreno-García, I., Delgado-Pardo, G., de Rey, C. C. V., Meneres-Sancho, S., \& Servera-Barceló, M. (2015). Neurofeedback, pharmacological treatment and behavioral therapy in hyperactivity: Multilevel analysis of treatment effects on electroencephalography. International Journal of Clinical and Health Psychology, 15(3), 217-25. [DOI:10.1016/j.ijchp.2015.04.003] [PMID] [PMCID] 
Moriyama, T. S., Polanczyk, G., Caye, A., Banaschewski, T., Brandeis, D., \& Rohde, L. A. (2012). Evidence-based information on the clinical use of neurofeedback for ADHD. Neurotherapeutics, 9(3), 588-98. [DOI:10.1007/s13311-012-0136-7] [PMID] [PMCID]

Morton, W. A., \& Stockton, G. G. (2000). Methylphenidate abuse and psychiatric side effects. Primary Care Companion to the Journal of Clinical Psychiatry, 2(5), 159-64. [DOI:10.4088/PCC. v02n0502] [PMID] [PMCID]

Murphy, S. M., Mcpherson, S., \& Robinson, K. (2014). Non-medical prescription opioid use and violent behaviour among adolescents. Journal of Child \& Adolescent Mental Health, 26(1), 35-47. [DOI:10.2989/17280583.2013.849607] [PMID]

Nelson, R. J., \& Trainor, B. C. (2007). Neural mechanisms of aggression. Nature Reviews Neuroscience, 8(7), 536-46. [DOI:10.1038/nrn2174] [PMID]

Patton, J. H., Stanford, M. S., \& Barratt, E. S. (1995). Factor structure of the Barratt impulsiveness scale. Journal of Clinical Psychology, 51(6), 768-74. https://onlinelibrary.wiley. com/doi/abs/10.1002/1097-4679(199511)51:6\%3C768::AIDJCLP2270510607\%3E3.0.CO;2-1

Pendar, R., Azarmehr, R., Shokrpour, N., Taghinezhad, A., Azadikhah, M., \& Nourinezhad, H. (2018). Comparing the effect of neurofeedback and verbal self-instruction on children afflicted with attention-deficit hyperactivity disorder: A cognitive-behavioral approach. Brain Broad Research in Artificial Intelligence and Neuroscience, 9(2), 128-38. https://lumenpublishing.com/journals/index.php/brain/article/view/2039

Polanczyk, G. V., Salum, G. A., Sugaya, L. S., Caye, A., \& Rohde, L. A. (2015). Annual research review: A meta-analysis of the worldwide prevalence of mental disorders in children and adolescents. The Journal of Child Psychology and Psychiatry, 56(3), 345-65. [DOI:10.1111/jcpp.12381] [PMID]

Puiu, A. A., Wudarczyk, O., Goerlich, K. S., Votinov, M., Herpertz-Dahlmann, B., \& Turetsky, B., et al. (2018). Impulsive aggression and response inhibition in attention-deficit/hyperactivity disorder and disruptive behavioral disorders: Findings from a systematic review. Neuroscience $\mathcal{E}$ Biobehavioral Reviews, 90, 231-46. [DOI:10.1016/j.neubiorev.2018.04.016] [PMID]

Sarvghadi, P., Ghaffari, A., \& Rostami, H. R. (2019). The effects of neurofeedback training on short-term memory and quality of life in women with breast cancer. International Journal of Therapy and Rehabilitation, 26(11), 1-8. [DOI:10.12968/ijtr.2018.0088]

Saylor, K. E., \& Amann, B. H. (2016). Impulsive aggression as a comorbidity of attention-deficit/hyperactivity disorder in children and adolescents. Journal of Child and Adolescent Psychopharmacology, 26(1), 19-25. [DOI:10.1089/cap.2015.0126] [PMID] [PMCID]

Shanshan, L., \& Zichao, Ch. (2017). Effects of neurofeedback training on dyslexic students' aggression: An experimental study. NeuroQuantology, 15(2), 269-76. [DOI:10.14704/ nq.2017.15.2.1072]

Sherlin, L. H., Arns, M., Lubar, J., Heinrich, H., Kerson, C., \& Strehl, U., et al. (2011). Neurofeedback and basic learning theory: Implications for research and practice. Journal of Neurotherapy, 15(4), 292-304. [DOI:10.1080/10874208.2011.623089]

Shokhmgar, Z., Sanagoo, A., Mohammadpour, M., Khaleghi, H., Ahangi, A., \& Mehrandish, N., et al. (2019). Comparison of the effectiveness of cognitive-behavioral therapy and neurofeedback for reducing symptoms of anxiety. Journal of Clinical and Basic Research, 3(2), 1-7. [DOI:10.29252/jcbr.3.2.1]

Singer, W. (2018). Neuronal oscillations: Unavoidable and useful? European Journal of Neuroscience, 48(7), 2389-98. [DOI:10.1111/ejn.13796] [PMID]

Sperry, S. H., Lynam, D. R., Walsh, M. A., Horton, L. E., \& Kwapil, T. R. (2016). Examining the multidimensional structure of impulsivity in daily life. Personality and Individual Differences, 94, 153-8. [DOI:10.1016/j.paid.2016.01.018]

Sterman, M. B., \& Egner, T. (2006). Foundation and practice of neurofeedback for the treatment of epilepsy. Applied Psychophysiology and Biofeedback, 31(1), 21. [DOI:10.1007/s10484-0069002-x] [PMID]

van Dongen-Boomsma, M., Vollebregt, M. A., Slaats-Willemse, D., \& Buitelaar, J. K. (2013). A randomized placebo-controlled trial of Electroencephalographic (EEG) neurofeedback in children with attention-deficit/hyperactivity disorder. The Journal of Clinical Psychiatry, 74(8), 821-7. [DOI:10.4088/ JCP.12m08321] [PMID]

Walker, J. (2013). QEEG-guided neurofeedback for anger/anger control disorder. Journal of Neurotherapy, 17(1), 88-92. [DOI:10. 1080/10874208.2012.705767]

Wilens, T. E., \& Spencer, T. J. (2010). Understanding attentiondeficit/hyperactivity disorder from childhood to adulthood. Postgraduate Medicine, 122(5), 97-109. [DOI:10.3810/ pgm.2010.09.2206] [PMID] [PMCID]

Wilson, V. E., Peper, E., \& Moss, D. (2006). “The mind room” in Italian soccer training: The use of biofeedback and neurofeedback for optimum performance. Biofeedback, 34(3), 79-81. https://www.proquest.com/openview/7681c400fdc0f3ad5 d413f99f5aba75e/1

Yadollahpour, A., Naraghi-Araani, M., \& Rashidi, S. (2015). Current advances in neurofeedback techniques for the treatment of ADHD. Biomedical \& Pharmacology Journal, 8, 165-77. https:// biomedpharmajournal.org/vol8marchspledition/ current-advances-in-neurofeedback-techniques-for-the-treatment-of-adhd/

Zucchetti, G., Ortega, E., Scholte, R. H. J., \& Rabaglietti, E. (2015) The mediating role of aggressive behaviour, emotional and behavioural instability on the association between ADHD symptoms and best friend conflicts. Current Psychology, 34(1), 97-111. [DOI:10.1007/s12144-014-9243-4] 
This Page Intentionally Left Blank 